Military Technical College Kobry El-Kobbah, Cairo, Egypt.

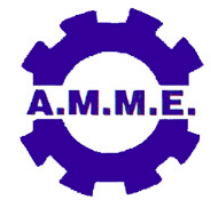

$17^{\text {th }}$ International Conference on Applied Mechanics and Mechanical Engineering.

\title{
LEADER-FOLLOWING CONSENSUS FOR A CLASS OF LINEAR MULTI-AGENT SYSTEMS UNDER SWITCHING NETWORKS
}

J. Huang*

\begin{abstract}
The existing result on the leader-following consensus problem for linear discrete-time multi-agent systems subject to switching networks is limited to undirected networks. In this paper, we further study the same problem for a class of linear multi-agent systems subject to directed switching networks. It is shown that, under certain assumptions, it is possible to solve the problem for directed networks by a distributed state feedback control law. Our approach is illustrated by a case study.
\end{abstract}

\section{KEY WORDS}

Cooperative control, Directed switching networks, Multi-agent systems, Discrete-time systems, Frequently connected.

* Professor, The Research Institute of Shenzhen, and Department of Mechanical and Automation Engineering, The Chinese University of Hong Kong, Shatin, Hong Kong, China. 


\section{INTRODUCTION}

Over the past decade, there has been extensive interest in consensus problem of both continuous-time and discrete-time multi-agent systems, see, for example, the survey paper [7] and the books [1], [10]. The leaderless consensus problem aims to make the states of all agents synchronize to a same trajectory, while the leader-following consensus problem requires the states of all agents to track a desired trajectory which is generated by the leader system. What makes the consensus problem interesting is that the problem has to be solved by a control law that satisfies certain communication constraints. Such a control law is called distributed control law. The communication constraints are described by a digraph witch can be static or time-varying. The most common time-varying graph is the so-called switching graph.

Under switching communication constraints, the consensus problem for linear discretetime multi-agent systems was mainly studied for multiple single-integrator systems [1], [4], [5], [6], [9]. An exception is [12] where both the leader-following and leaderless consensus problems were studied for a class of general linear discrete-time multiagent systems under the assumption that the switching graph is undirected and satisfies the jointly connected condition. The jointly connected condition is perhaps the mildest condition on a switching graph as it allows the graph to be disconnected at every time instant. Nevertheless, the assumption that the graph is undirected is restrictive in practice. In this paper, we will study the leader-following consensus problem for the same class of systems studied in [12] without assuming that the graph is undirected. Our investigation shows that, for some interesting cases, the consensus problem for the system studied in [12] is solvable even if the switching graph is not undirected and the graph is disconnected at almost every switching time instant.

The rest of this paper is organized as follows: In Section II, we introduce the leaderfollowing consensus problem. In Section III, we present our main result. A case study is given to illustrate our design in Section IV. Finally, we conclude the paper in Section V.

The following notation will be used throughout this paper: $\otimes$ denotes the Kronecker product of matrices. Some properties of Kronecker product are useful in this paper: $(A \otimes B)^{T}=A^{T} \otimes B^{T},(A \otimes B)(C \otimes D)=(A C) \otimes(B D),(A+B) \otimes C=A \otimes C+B \otimes C$, $A \otimes(B+C)=A \otimes B+A \otimes C . \mathbb{Z}^{+}$denotes the set of nonnegative integers. $\mathbf{1}_{N}$ denotes an $N \times 1$ column vector whose elements are all 1 . Given the matrices $A_{i} \in$ $\mathbb{R}^{n_{i} \times m}$, we denote $\operatorname{col}\left(A_{1}, \ldots, A_{N}\right)=\left[A_{1}^{T}, \ldots, A_{N}^{T}\right]^{T}$. Let $\sigma: \mathbb{Z}^{+} \rightarrow \mathcal{P}$, where $\mathcal{P}=\{1,2, \ldots, \rho\}$. Throughout this paper, we assume $\sigma(\cdot)$ is a piecewise constant switching signal in the sense that there exists a subsequence $\left\{k_{i}\right\}$ of $\{k\}$, called switching instants, such that $\sigma(k)$ is a constant for $k_{i} \leq k<k_{i+1}$ for any $k_{i} \geq 0$.

\section{PROBLEM STATEMENT}

Like in [12], we consider the leader-following consensus problem for the following class of linear discrete-time multi-agent systems subject to a switching network topology:

$$
x_{i}(k+1)=A x_{i}(k)+B u_{i}(k), \quad i=1, \ldots, N, \quad k=0,1, \ldots
$$


where $x_{i}(k) \in \mathbb{R}^{n}$ and $u_{i}(k) \in \mathbb{R}^{m}$ are the state and control of the agent $i$, respectively, and $(A, B)$ is controllable. Roughly, our objective is to design a distributed control law such that the solution of each subsystem of Eqn. (1) approaches some discrete-time signal $x_{0}(k)$ asymptotically. Also like in [12], we assume that the signal $x_{0}(k)$ is generated by a linear autonomous system of the following form:

$$
x_{0}(k+1)=A x_{0}(k)
$$

with an arbitrary initial state $x_{0}(0) \in \mathbb{R}^{n}$.

To handle our problem, we treat the system composed of system (2) and system (1) as a multi-agent system with Eqn. (1) as the leader system and Eqn. (2) as the follower system, respectively. Associated with system (1), system (2) and the given piecewise constant switching signal $\sigma(\cdot)$, we can define a dynamic graph $\overline{\mathcal{G}}_{\sigma(k)}=\left(\overline{\mathcal{V}}, \bar{\varepsilon}_{\sigma(k)}\right)$ where $\overline{\mathcal{V}}=\{0,1, \ldots, N\}$ and $(j, i) \in \overline{\mathcal{E}}_{\sigma(k)}, i=1, \ldots, N, j=0,1, \ldots, N$, if and only if the control $u_{i}$ can access $x_{i}-x_{j}$ at time $k$. To introduce our control law, for any $k \geq 0$, let $\overline{\mathcal{L}}_{\sigma(k)}=$ $\left[l_{i j}(k)\right] \in \mathbb{R}^{(N+1) \times(N+1)}, i, j=0,1, \ldots, N$, be the Laplacian of the graph $\overline{\mathcal{G}}_{\sigma(k)}$. Then

$$
u_{i}(k)=K \sum_{j=0}^{N} l_{i j}(k)\left(x_{i}(k)-x_{j}(k)\right), \quad i=1, \ldots, N
$$

where $K \in \mathbb{R}^{\mathrm{m} \times \mathrm{n}}$ is the gain matrix to be defined later.

Definition 2.1 (Leader-Following Consensus Problem): Given the leader system represented by Eqn. (2), the follower system represented by Eqn. (1) and the associated dynamic graph $\overline{\mathcal{G}}_{\sigma(k)}$, find the gain matrix $K$ such that the closed-loop system composed of the plant (1) and the state feedback protocol (3) has the property that, for any initial condition and for $i=1, \ldots, N,\left(x_{i}(k)-x_{0}(k)\right) \rightarrow 0$ as $k \rightarrow \infty$.

Remark 2.1: Let $\mathcal{G}_{\sigma(k)}=\left(\mathcal{V}, \mathcal{E}_{\sigma(k)}\right)$ be a sub-graph of $\overline{\mathcal{G}}_{\sigma(k)}$, where $\mathcal{V}=\{1, \ldots, N\}$ and $\mathcal{E}_{\sigma(k)} \subseteq \mathcal{V} \times \mathcal{V}$ is obtained from $\overline{\mathcal{E}}_{\sigma(k)}$ by removing all the edges between the node 0 and the nodes in $\mathcal{V}$. Let $\Delta_{\sigma(k)}$ be an $N \times N$ diagonal matrix with $-l_{i 0}(k)$ as its $i$ th diagonal element, and let $\mathcal{L}_{\sigma(k)}$ be the Laplacian of $\mathcal{G}_{\sigma(k)}$. Then, it can be verified that

$$
\overline{\mathcal{L}}_{\sigma(k)}=\left[\begin{array}{cc}
0_{1 \times 1} & 0_{1 \times N} \\
-\Delta_{\sigma(k)} \mathbf{1}_{N} & \mathcal{L}_{\sigma(k)}+\Delta_{\sigma(k)}
\end{array}\right]
$$

Let $H_{\sigma(k)}=\mathcal{L}_{\sigma(k)}+\Delta_{\sigma(k)}$. Then, none of the eigenvalues of $H_{\sigma(k)}$ have negative real parts. Moreover, if the graph $\overline{\mathcal{G}}_{\sigma(k)}$ is connected, then all the eigenvalues of $H_{\sigma(k)}$ have positive real parts [3].

In this paper, we make the following assumption on the matrices $A$ and $B$. 
Assumption 2.1: The system matrix $A$ is neutrally stable, i.e., all eigenvalues of $A$ are semi-simple ${ }^{1}$ with modulus 1 , and the matrix $B$ is nonsingular.

Remark 2.2: The system matrix $A$ is said to be marginally stable if all the eigenvalues of $A$ are inside the unit circle, and those Eigen values with modulus 1 are semi-simple. Using the same argument as we used in [12], Assumption 2.1 can be relaxed to the case where the matrix $A$ is marginally stable.

Remark 2.3: Under Assumption 2.1, we can assume the Jordan form $\bar{A}$ of $A$ takes the following form,

$$
\bar{A}=\text { blockdiag }\left\{\left[\begin{array}{cc}
\alpha_{1} & -\beta_{1} \\
\beta_{1} & \alpha_{1}
\end{array}\right], \ldots,\left[\begin{array}{cc}
\alpha_{r} & -\beta_{r} \\
\beta_{r} & \alpha_{r}
\end{array}\right], I_{s-2 r},-I_{n-s}\right\}
$$

where $0 \leq 2 \mathrm{r} \leq \mathrm{s} \leq \mathrm{n}$, and, for $k=1, \ldots, r, \alpha_{k}^{2}+\beta_{k}^{2}=1$. Clearly, $\bar{A}$ is an orthogonal matrix.

By Remark 2.3, there exists a nonsingular real matrix $P$ such that

$$
A=P^{-1} \bar{A} P
$$

Under the transformation $\bar{x}_{i}=P x_{i}$, system (1) is transformed into

$$
\bar{x}_{i}(k+1)=\bar{A} \bar{x}_{i}(k)+\bar{B} u_{i}(k), \quad i=1, \ldots N
$$

where $\bar{B}=P B$. Under Assumption 2.1, without loss of generality, we can assume that $\bar{B}$ is an identity matrix.

\section{MAIN RESULT}

We begin this section with the following two assumptions.

Assumption 3.1: For all $p=1, \ldots, \rho$, there exists a $\hat{\mu}>0$ such that the matrix $H_{p}^{T}+$ $H_{p}-\hat{\mu} H_{p}^{T} H_{p} \geq 0$.

Assumption 3.2: There exists a subsequence $\left\{t_{j}\right\}$ of $\{k\}$ satisfying $\lim _{j \rightarrow \infty} t_{j}=\infty$ such that the node 0 can reach every other node of the graph $\overline{\mathcal{G}}_{\sigma\left(t_{j}\right)}$.

Remark 3.1: If the graph $\mathcal{G}_{\sigma(k)}$ is undirected, then the matrix $H_{\sigma(k)}$ is symmetric and positive semi-definite. In this case, denote the eigenvalues of $H_{p}$ by $\lambda_{i}(p), \mathrm{i}=1, \ldots N$. Let $\lambda_{M}(p)$ and $\lambda_{m}(p)$ denote the largest and the smallest nonzero eigenvalues of the matrix $H_{p}$. Also let $\lambda_{M}=\max _{p=1, \ldots, \rho}\left\{\lambda_{M}(p)\right\}$ and $\lambda_{m}=\min _{p=1, \ldots, \rho}\left\{\lambda_{m}(p), \lambda_{m}(p) \neq 0\right\}$. Since, for any $0 \leq p \leq \rho$, the eigenvalues of $H_{p}^{T}+H_{p}-\mu H_{p}^{T} H_{p}$ are given by $2 \lambda_{i}(p)-$

\footnotetext{
${ }^{1}$ An eigenvalue of a matrix is semi-simple if all the Jordan blocks associated with this eigenvalue have dimension one.
} 
$\mu \lambda_{i}(p)^{2}$, Assumption 3.1 is satisfied with $\hat{\mu}=\frac{2 \lambda_{m}}{\lambda_{M}{ }^{2}}$. However, what makes Assumption 3.1 interesting is that, for many cases, even if the graph $\mathcal{G}_{\sigma(k)}$ is not undirected, there exists $\hat{\mu}>0$ such that Assumption 3.1 is satisfied. This is the case whenever, for any $p=1, \ldots, \rho$, the matrix $H_{p}^{T}+H_{p}$ is positive definite even though $H_{p}$ are not all symmetric. For example, let $H_{p}=\left[\begin{array}{cc}a_{p} & 1 \\ 0 & a_{p}\end{array}\right]$ where $a_{p}>\frac{1}{2}$. Then it can be verified that $H_{p}^{T}+H_{p}$ is positive definite. In fact, even if $H_{p}^{T}+H_{p}$ is not positive definite, there still exists $\hat{\mu}>0$ such that the matrix $H_{p}^{T}+H_{p}-\mu H_{p}^{T} H_{p}$ is positive semi-definite for all $0<$ $\mu<\hat{\mu}$. For example, none of the matrices $H_{p}, p=1,2,3$, in the example of next section are nonsingular, yet they still satisfy Assumption 3.1. By Remark 2.1, Assumption 3.2 implies that there exists a subsequence $\left\{t_{j}\right\}$ of $\{k\}$ such that the matrices $H_{\sigma\left(t_{j}\right)}$ are nonsingular for all $t_{j}$. Assumption 3.2 is interesting because it does not require the graph $\mathcal{G}_{\sigma(k)}$ to be connected at every time. For convenience, when the dynamic graph $\mathcal{G}_{\sigma(k)}$ satisfies Assumption 3.2, we say the graph $\mathcal{G}_{\sigma(k)}$ is frequently connected.

Lemma 3.1: Under Assumptions 3.1 and 3.2, let the matrix $\bar{A}$ be as defined in Eqn. (4). Then, the origin of the following linear discrete-time switched system

$$
\xi(k+1)=\left(I_{N} \otimes \bar{A}-\mu H_{\sigma(k)} \otimes \bar{A}\right) \xi(k)
$$

is asymptotically stable for all $\mu$ satisfying $0<\mu<\hat{\mu}$.

Proof: Let

$$
V(\xi(k))=\frac{1}{2} \xi^{T}(k) \xi(k)
$$

Then the difference of Eqn. (8) along the system represented by Eqn. (7) satisfies

$$
\begin{aligned}
\left.\Delta V(\xi(k))\right|_{(7)} & =\xi^{T}(k+1) \xi(k+1)-\xi^{T}(k) \xi(k) \\
& =-\xi^{T}(k)\left(\mu\left(H_{\sigma(k)} \otimes I_{N}+H_{\sigma(k)}^{T} \otimes I_{N}\right)-\mu^{2} H_{\sigma(k)}^{T} H_{\sigma(k)} \otimes I_{N}\right) \xi(k)
\end{aligned}
$$

By Assumption 3.1, we have

$$
\left.\Delta V(\xi(k))\right|_{(7)}=-\epsilon \mu \xi^{T}(k)\left(H_{\sigma(k)}^{T} H_{\sigma(k)} \otimes I_{N}\right) \xi(k) \leq 0
$$

where $\epsilon=(\hat{\mu}-\mu)$.

Thus, $\lim _{k \rightarrow \infty} V(\xi(k))$ exists since $V(\xi(k))$ is non-increasing and lower bounded. By Assumption 3.2 and Remark 3.1, $H_{\sigma\left(t_{j}\right)}$ is nonsingular. Thus, $H_{\sigma\left(t_{j}\right)}^{T} H_{\sigma\left(t_{j}\right)}$ is positive definite. It follows from Eqn. (10) that, for all $j \geq 0$,

$$
\left.\Delta V\left(\xi\left(t_{j}\right)\right)\right|_{(7)}=-\epsilon \mu \xi^{T}\left(t_{j}\right)\left(H_{\sigma\left(t_{j}\right)}^{T} H_{\sigma\left(t_{j}\right)} \otimes I_{N}\right) \xi\left(t_{j}\right)
$$


Thus, $\lim _{j \rightarrow \infty} \xi\left(t_{j}\right)=0$. Therefore, $\lim _{k \rightarrow \infty} \xi(k)=0$.

Theorem 3.1: Under Assumptions 2.1, 3.1, and 3.2, the leader-following consensus problem for the leader system represented by Eqn. (2) and the follower system represented by Eqn. (1) is solvable by the distributed state feedback protocol (3) with the gain matrix $K=\mu P^{T} P A$, where $P$ is defined in Eqn. (5) and $0<\mu<\hat{\mu}$.

Proof: Under Eqn. (3), the closed-loop system of agent $i$ is

$$
x_{i}(k+1)=\mathrm{A} x_{i}(k)+\mu P^{T} P A \sum_{j=0}^{N} l_{i j}(k)\left(x_{i}(k)-x_{j}(k)\right)
$$

Let $\bar{w}_{i}(k)=P\left(x_{i}(k)-x_{0}(k)\right)$. Then by Eqn. (2) and Eqn. (12), we have

$$
\bar{w}_{i}(k+1)=\bar{A} \bar{w}_{i}(k)+\mu \bar{A} \sum_{j=0}^{N} l_{i j}(k)\left(\bar{w}_{i}(k)-\bar{w}_{j}(k)\right)
$$

where $\bar{w}_{0}(k):=0$. Then system represented by Eqn. (13) can be put into the following compact form

$$
\bar{w}(k+1)=\left(I_{N} \otimes \bar{A}-\mu H_{\sigma(k)} \otimes \bar{A}\right) \bar{w}(k)
$$

where $\bar{w}(k)=\operatorname{col}\left(\bar{w}_{1}(k), \ldots, \bar{w}_{N}(k)\right)$. System (14) is in the form of Eqn. (7) with $\xi(k)=$ $\bar{w}(k)$. Since the system (14) satisfies all the conditions of Lemma 3.1, by Lemma 3.1, $\lim _{k \rightarrow \infty} \bar{w}(k)=0$. Thus, for all $i=1, \ldots, N, x_{i}(k)$ asymptotically converge to $x_{0}(k)$.

\section{A CASE STUDY}

Consider the leader system represented by Eqn. (2) and the follower system represented by Eqn. (1) with $N=4, B=I_{2}$, and

$$
A=\left[\begin{array}{cc}
\cos 1 & \sin 1 \\
-\sin 1 & \cos 1
\end{array}\right]
$$

Assume the communication graph is dictated by the following switching signal:

$$
\sigma(t)=\left\{\begin{array}{rr}
1, & \text { if } s T_{0} \leq t<\left(s+\frac{1}{4}\right) T_{0} \\
2, & \text { if }\left(s+\frac{1}{4}\right) T_{0} \leq t<\left(s+\frac{1}{2}\right) T_{0} \\
3, & \text { if }\left(s+\frac{1}{2}\right) T_{0} \leq t<\left(s+\frac{3}{4}\right) T_{0} \\
4, & \text { if }\left(s+\frac{3}{4}\right) T_{0} \leq t<(s+1) T_{0}
\end{array}\right.
$$


where $T_{0}=2, s=0,1,2, \ldots$. The four digraphs $\overline{\mathcal{G}}_{i}, i=1,2,3,4$, are described by Fig. 1 where the node 0 is associated with the leader and the other nodes are associated with the followers. The four matrices associated with the four digraphs $\overline{\mathcal{G}}_{i}, i=1,2,3,4$, are

$$
\begin{array}{lll}
H_{1}=\left[\begin{array}{llll}
1 & 0 & 0 & 0 \\
0 & 0 & 0 & 0 \\
0 & 0 & 0 & 0 \\
0 & 0 & 0 & 0
\end{array}\right], & H_{2}=\left[\begin{array}{cccc}
0 & 0 & 0 & 0 \\
0 & 1 & 0 & 0 \\
0 & -1 & 1 & 0 \\
0 & 0 & 0 & 0
\end{array}\right] \\
H_{3}=\left[\begin{array}{llll}
1 & 0 & 0 & 0 \\
0 & 0 & 0 & 0 \\
0 & 0 & 0 & 0 \\
-1 & 0 & 0 & 1
\end{array}\right], & H_{4}=\left[\begin{array}{cccc}
1 & 0 & 0 & 0 \\
0 & 2 & -1 & 0 \\
-1 & 0 & 1 & 0 \\
-1 & 0 & 0 & 1
\end{array}\right]
\end{array}
$$

It can be seen that none of the matrices $H_{i}, i=1,2,3$, are nonsingular, yet $H_{1}^{T}+H_{1}-$ $\mu H_{1}^{T} H_{1}$ is positive semi-definite for $0<\mu<2$, and, for $i=2,3, H_{i}^{T}+H_{i}-\mu H_{i}^{T} H_{i}$ are positive semi-definite for $0<\mu<1$. Finally, $H_{4}$ is nonsingular and $H_{4}^{T}+H_{4}-\mu H_{4}^{T} H_{4}$ is positive definite for $0<\mu<0.52$. By Theorem 3.1 and Remark 3.1, the consensus problem for the leader system represented by Eqn. (2) and the follower system represented by Eqn. (1) can be solved by the control law of the form (3) with $0<\mu<$ 0.52 . The simulation result is shown in Fig. 2 .

\section{CONCLUSIONS}

In the literature such as [6] and [12], the leader-following consensus problem for discrete-time linear systems subject to switching networks have been studied under the assumption that the network is undirected. In this paper, we have further studied the leader-following consensus problem for a class of discrete-time linear systems under directed switching network topology. By establishing a stability result on a class of linear discrete-time switched systems satisfying frequently connected condition, we have shown that, for some interesting cases, the consensus problems can be solved via state feedback protocols even though the graph is not undirected.

\section{ACKNOWLEDGEMENTS}

This work has been supported in part by the Research Grants Council of the Hong Kong Special Administration Region under grant No. 412813, and in part by the National Natural Science Foundation of China under grant No. 61174049.

\section{REFERENCES}

[1] D. P. Bertsekas and J. N. Tsitsiklis (1989). Parallel and Distributed Computation: Numerical Methods. Englewood Cliffs, NJ:Prentice Hall. 
[2] C. Godsil and G. Royle (2001). Algebraic Graph Theory. New York: SpringerVerlag.

[3] J. Hu and Y. Hong (2007). Leader-following coordination of multi-agent systems with coupling time delays', Physica A: Statistical Mechanics and its Applications, 374(2), pp. 853-863.

[4] A. Jadbabaie, J. Lin, and A. S. Morse (2003). Coordination of groups of mobile agents using nearest neighbor rules. IEEE Transactions on Automatic Control, 48(6), 988-1001.

[5] A. Nedi'c, A. Olshevsky, A. Ozdaglar, and J.N. Tsitsiklis (2009). On distributed averaging algorithms and quantization effects. IEEE Transactions on Automatic Control, 54(11), 2506-2616.

[6] R. Olfati-Saber and R. M. Murray (2004). Consensus problems in networks of agents with switching topology and time-delays. IEEE Transactions on Automatic Control, 49(9), 1520-1533.

[7] R. Olfati-Saber, J. A. Fax, and R. M. Murray (2007). Consensus and cooperation in networked multi-agent systems. Proceedings of the IEEE, 95(1), 215-233.

[8] W. Ren (2008b). Synchronization of coupled harmonic oscillators with local interaction. Automatica, 44(2), 3195-3200.

[9] W. Ren and R.W. Beard (2005). Consensus seeking in multiagent systems under dynamically changing interaction topologies. IEEE Transactions on Automatic Control, 50(5), 655-661.

[10] W. Ren and R. W. Beard (2008). Distributed Consensus in Multi-vehicle Cooperative Control. Communications and Control Engineering Series, London: Springer-Verlag.

[11] Y. Su and J. Huang (2012). Solvability of Two Consensus Problems for a Class of Linear Multi-Agent Systems. IEEE Transactions on Automatic Control, to appear.

[12] Y. Su and J. Huang (2012). Two consensus problems for discrete-time multiagent systems with switching topology. Automatica, 46(10), 1988-1997. 


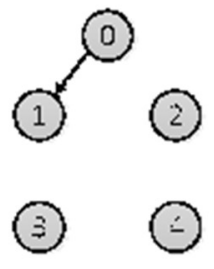

(a) $\mathcal{G}_{1}$

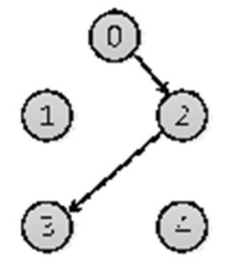

(b) $\mathcal{G}_{2}$

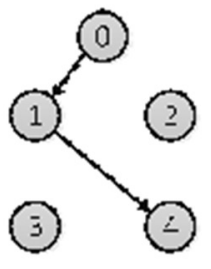

(c) $\mathcal{G}_{3}$

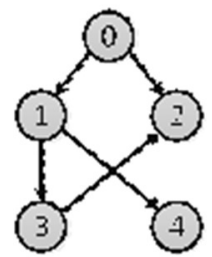

(d) $\mathcal{G}_{4}$

Fig.1. Switching topology $\overline{\mathcal{G}}_{\sigma(k)}$ with $\mathcal{P}=\{1,2,3,4\}$
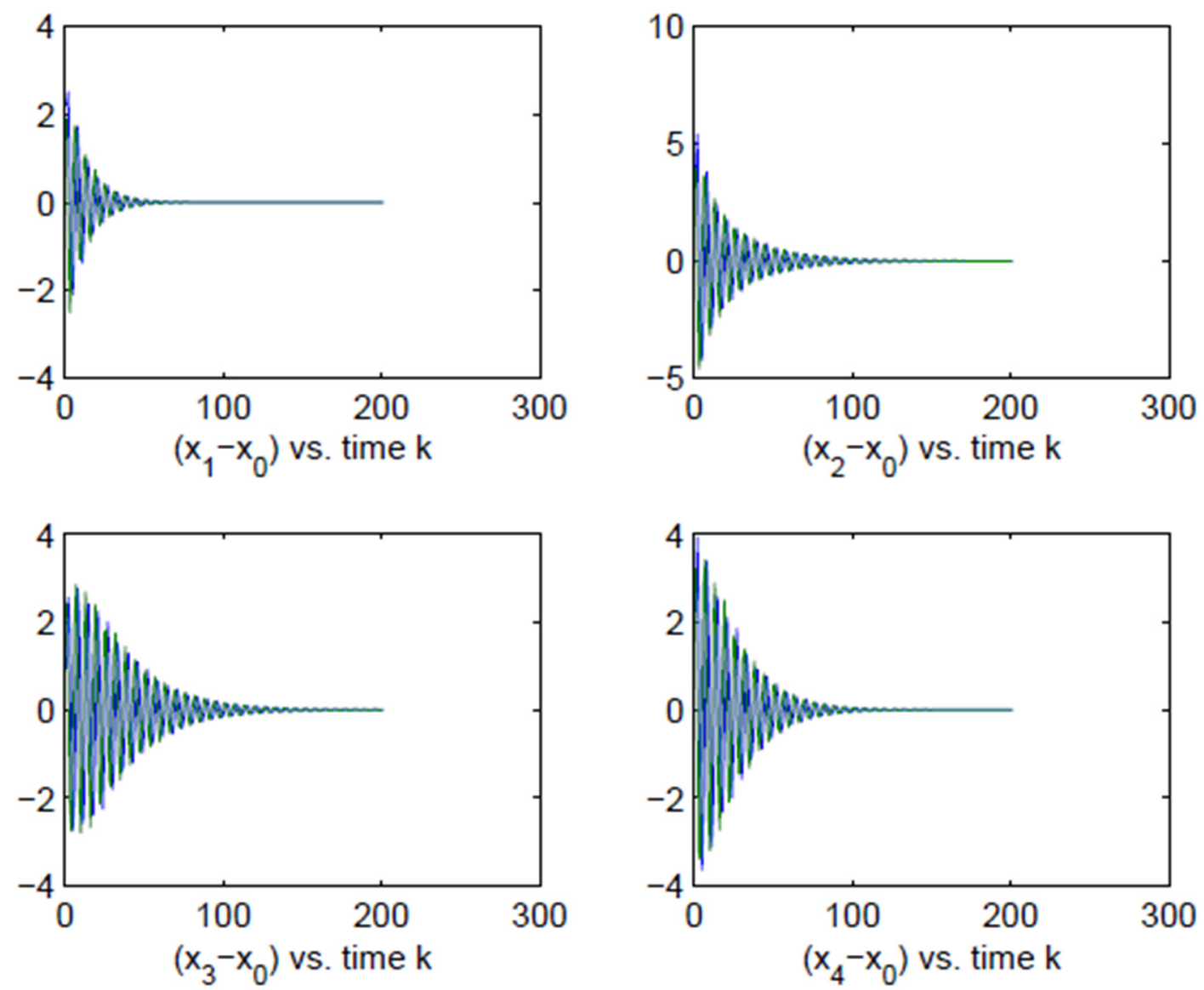

Fig.2. Profile of $\left(x_{i}-x_{0}\right), i=1,2,3,4$. 


\section{APPENDIX A}

\section{Digraph}

We first introduce some graph notation which can be found in [2]. A digraph $\mathcal{G}=(\mathcal{V}, \mathcal{E})$ consists of a finite set of nodes $\mathcal{V}=\{1, \ldots, N\}$ and an edge set $\mathcal{E}=\{(i, j), i, j \in \mathcal{V}, i \neq j\}$. A node $i$ is called a neighbor of a node $j$ if the edge $(i, j) \in \mathcal{E}$. $\mathcal{N}_{i}$ denotes the subset of $\mathcal{V}$ that consists of all the neighbors of the node $i$. If the graph $\mathcal{G}$ contains a sequence of edges of the form $\left(i_{1}, i_{2}\right),\left(i_{2}, i_{2}\right), \ldots,\left(i_{k}, i_{k+1}\right)$, then the set $\left\{\left(i_{1}, i_{2}\right),\left(i_{2}, i_{2}\right), \ldots,\left(i_{k}, i_{k+1}\right)\right\}$ is called a path of $\mathcal{G}$ from $i_{1}$ to $i_{k+1}$, and the node $i_{k+1}$ is said to be reachable from node $i_{1}$. The edge $(i, j)$ is called undirected if $(i, j) \in \mathcal{E}$ implies $(j, i) \in \mathcal{E}$. The graph is called undirected if every edge in $\mathcal{E}$ is undirected. $A$ graph is called connected if there exists a node $i$ such that any other nodes are reachable from node $i$. A digraph $\mathcal{G}_{s}=\left(\mathcal{V}_{s}, \mathcal{E}_{s}\right)$ is a subgraph of $\mathcal{G}=(\mathcal{V}, \mathcal{E})$ if $\mathcal{V}_{s} \subseteq \mathcal{V}$ and $\varepsilon_{s} \subseteq \mathcal{E} \cap\left(\mathcal{V}_{s} \times \mathcal{V}_{s}\right)$.

A matrix $\mathcal{A}=\left[a_{i j}\right] \in \mathbb{R}^{N \times N}$ is called the weighted adjacency matrix of $\mathcal{G}$ if it satisfies $a_{i i}=0$, and, for $i \neq j, a_{i j}>0 \Leftrightarrow(j, i) \in \mathcal{E}$ and $a_{i j}=a_{j i}$ if $(i, j) \in \mathcal{E}$ is undirected. The Laplacian of $\mathcal{G}$ is defined as $\mathcal{L}=\left[l_{i j}\right] \in \mathbb{R}^{N \times N}$, where $l_{i i}=\sum_{j=1}^{N} a_{i j}, l_{i j}=-a_{i j}$ for $i \neq j$. Since the Laplacian $\mathcal{L}$ is of zero row sum, $\mathcal{L} \mathbf{1}_{N}=0$. 